dent of the choice of $w$ provided we choose $w$ great enough, say $w \geqslant 16$. A change in $S$ will basically move the curves only up and down; it will not affect the positions of their minima.

We notice again that increasing the bit size will decrease the optimal choice of $\alpha$. Comparing Figs. 6 and 10 we see that content addressable memories should have smaller branching ratios than random-access memories. For $b_{1}=4$, which seems a reasonable figure, the optimal choice of $\alpha$ is 4 .

\section{Conclusion}

We have presented a general method for analyzing the cost and performance of recursively defined VLSI structures. Parameters of any such structure may be optimized with respect to time, area, or some combination of the two. While we have chosen the area-time product, it is clear that some other choice may be appropriate for any given application.

The results of this study indicate that as more processing is available in each module at level zero, the optimal value of $\alpha$ will decrease. A system with $\alpha=4$ would seem to be appropriate for memories in which substantial processing is comingled with storage.

Very general arguments were used to generate the basic recursive structure. For that reason it appears that a very large fraction of VLSI computing structures will be designed in this way. We have discussed two examples, one in which the basic elements were bits of storage, and one with words of storage at the lowest level. They gave rise to rather different recursive structures. The way in which their area and time measures were established should make it clear how to apply these techniques to other recursively defined computing structures.

Carver A. Mead, for a biography and photograph, see this issue, p. 470 .

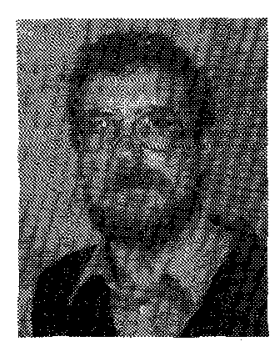

Martin Rem was born in The Netherlands on September 22, 1946. He received the B.S. degree in mathematics and physics and the M.S. degree in mathematics from the University of Amsterdam, Holland, in 1968 and 1971 . respectively, and the Ph.D. degree in computer science from Eindhoven University of Technology, Eindhoven, The Netherlands, in 1976.

$\mathrm{He}$ is an Associate Professor of Mathematics in the Department of Mathematics, Eindhoven University of Technology. He is presently a Visiting Professor of Computer Science in the Department of Computer Science, California Institute of Technology, Pasadena, CA. His major research interests are in the area of programming of machines with instore processing, semantics of programming languages, correctness proofs, and well-structured machine designs.

Dr. Rem is a member of the Association for Computing Machinery, the Dutch Computer Society NGI, and the Dutch Mathematical Society.

\title{
Delay-Time Optimization for Driving and Sensing of Signals on High-Capacitance Paths of VLSI Systems
}

\author{
AMR M. MOHSEN, MEMBER, IEEE, AND CARVER A. MEAD
}

\begin{abstract}
Transmission of signals on large capacitance paths in a VLSI system may result in substantial degradation of the overall system performance. In this paper minimization of the delay times associated with driving and sensing signals from large capacitance paths by optimizing the fan-out factor of the driver stages, the gain of the input sensing stages, and the path voltage swing are examined. Examples of driving signals on a high capacitance path with two driving schemes are: a push-pull depletion-load driver chain and a fixed driver; and of sensing signals with two sensing schemes: a single-ended depletion-load inverter input stage and a balanced regenerative strobed latch are presented. We conclude that minimum delay time is achieved when the delay times of the successive stages of the driver chain, the high capacitance path, and the input sensing stage are comparable.

A. M. Mohsen is with Intel Corporation, Santa Clara, CA, and the California Institute of Technology, Pasadena, CA 91125.

C. A. Mead is with the Department of Computer Science, California Institute of Technology, Pasadena, CA 91125.
\end{abstract}

In general, transmission time of signals in a system is minimized when the delay times of the different stages of the system are comparable.

\section{INTRODUCTION}

$T$ HE OVERALL PERFORMANCE of VLSI systems may be seriously degraded if signals need to be transmitted from one part to other parts in the system across large capacitance paths [1]. This large fan-out situation often occurs in the case of control drivers that are required to drive a large number of inputs to memory cells or logic-function blocks across a chip, or in the case of sensing stored information from small cells of large memory arrays. A similar and even more serious problem is driving wires which go off the silicon chip to other chips or input and output devices. In such cases, the 


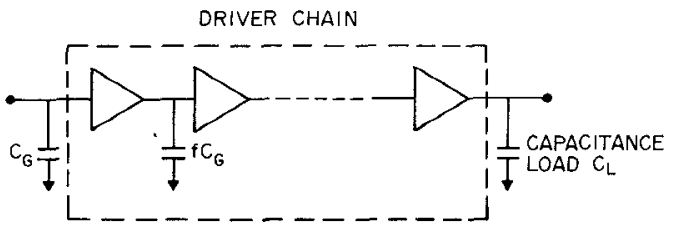

Fig. 1. Driver chain driving a high capacitance load $C_{L}$.

ratio of the capacitance that must be driven to the inherent capacitance of a gate circuit on the chip is often many orders of magnitude, causing a serious delay and degradation of system performance.

In this paper we examine, in general terms, optimum means of minimizing the delay time associated with transmitting information on large capacitance paths. In Section II, we analyze the driving of capacitive loads in the minimum possible time. In Section III, we examine the driving and sensing circuits with minimum possible delay times. In Section IV, we consider the sensing of signals on large capacitance lines driven by fixed sources. The general guidelines for designing the driver and sensing circuits of signals on high capacitance paths for minimum delay time are summarized in Section IV.

\section{Driving Large Capacitive Loads}

Consider how we may drive a capacitive load $C_{L}$ in the minimum possible time. Let us assume we are starting with a signal $V_{i}$ at the input of an elementary driver of input capacitance $C_{G}$. The elementary driver can be a simple static inverter or a dynamic clocked driver. Define the ratio of the load capacitance to the input capacitance $C_{L} / C_{G}$ as $Y$. It seems intuitively clear that the optimum way to drive a large capacitance is to use the elementary driver to drive a larger driver and that larger driver to drive a still larger driver until at some point the larger driver is able to drive the load capacitance directly, as shown in Fig. 1. Let the delay time associated with the elementary driver driving a similar driver be $\tau_{\mathrm{Dr}}$. Thus the delay associated with the elementary driver driving a larger driver by a factor $f$ is $f \tau_{\mathrm{Dr}}$. If $N$ such stages are used, each larger than the previous by a factor $f$, then the total delay of the driver chain $\tau_{\mathrm{ch}}$ is given by

$$
\tau_{\mathrm{ch}}=N f \tau_{\mathrm{Dr}} \text {. }
$$

Also, the capacitance ratio $Y$ is related to $N$ and $f$ by

$$
Y=f^{N}, \quad \ln Y=N \ln f .
$$

Substituting (2) into (1)

$$
\tau_{\mathrm{ch}}=\ln Y \cdot\left[\frac{f}{\ln f}\right] \tau_{\mathrm{Dr}} .
$$

Thus the total delay is always proportional to $\ln Y$ as a result of the exponential growth in successive stages of the driver. The multiplicative factor $f / \ln (f)$ is plotted as a function of $f$ in Fig. 2 normalized to its minimum value $e$. Total delay time is minimized when each stage is larger than the previous one by a factor of $e$, the base of natural logarithms. Minimum total delay $\tau_{\mathrm{ch} / \mathrm{min}}$ is given by

$$
\tau_{\mathrm{ch} / \min }=\tau_{\mathrm{Dr}} e \ln \left(\frac{C_{L}}{C_{G}}\right) .
$$

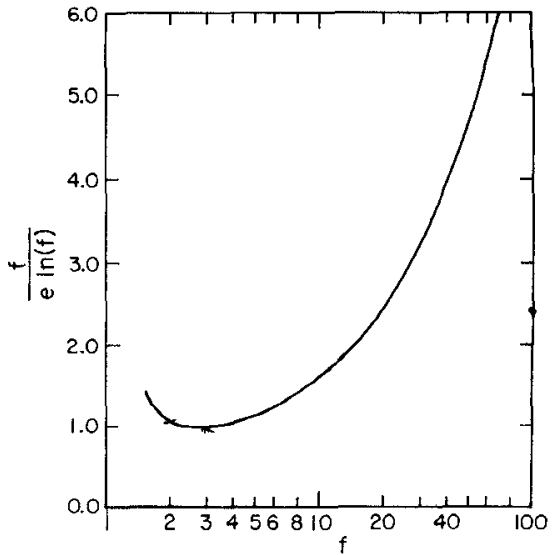

Fig. 2. Relative time penalty $(f / e \ln f)$ versus fan-out factor $f$.

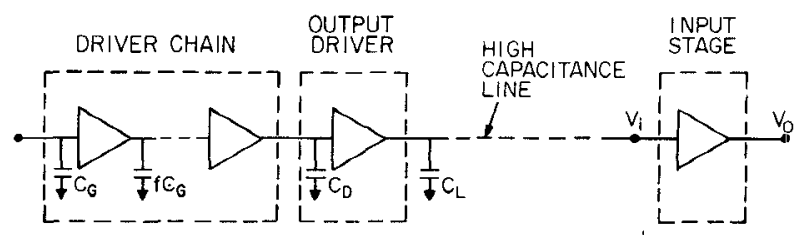

Fig. 3. Driver chain and output driver drives a high capacitance line $C_{L}$. Input stage receives input signal $V_{i}$ to generate output voltage $V_{o}$.

The minimum of the driver-chain delay in Fig. 2 is rather broad with a relatively small delay-time penalty for fan-out factor $f$ above $e$.

\section{Driving AND SEnsing Signals on Large CAPACITANCE LINES}

Consider how we may minimize the time to transfer a signal through a high capacitance line by optimizing the driver circuit at one end of the line and the input sensing circuit at the other end of the line. It has been shown previously that a driver chain can be optimized to minimize the delay time required to drive the line capacitance $C_{L}$. We will consider below the implications of optimizing the input sensing circuit with the driver circuit by examining the effect of the gain of the input stage and the line voltage swing on the total delay time of signal transmission on the high capacitance path.

In Fig. 3 the driver is made of a driver chain as described in Section II, where the voltage swing is equal to the supply voltage, and an output driver that drives the large capacitance line with a voltage swing equal to $V_{i}$. The input stage senses the signal at the other end of the line and generates an output voltage $V_{o}$ equal to the supply voltage. The input stage can be a single-ended circuit or a differential regenerative or nonregenerative circuit. The gain of the input stage $G$ is defined as

$$
G=\frac{V_{o}}{V_{i}}
$$

The delay associated with the input stage sensing $\tau_{i}$ is a function of the input voltage swing $V_{i}$ required to generate $V_{o}$ at the output, i.e., it is a function of the input-stage gain

$$
\text { Input-Stage Delay }=\tau_{i}\left(V_{i}\right) \text {. }
$$




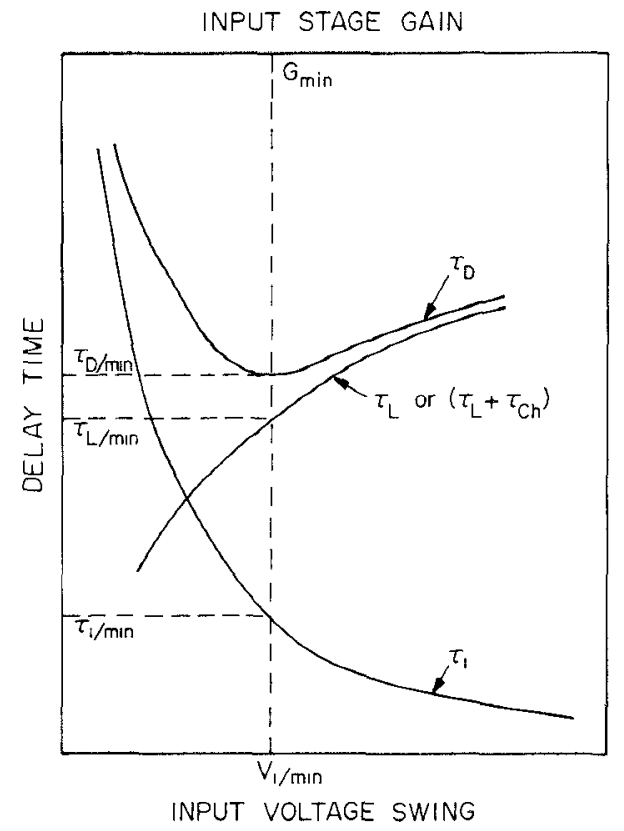

Fig. 4. Delay time of driver stages $\tau_{\mathrm{ch}}$, output driver $\tau_{L}$, and input stage $\tau_{i}$ versus input-stage gain and input voltage swing.

For smaller $V_{i}$, the input stage sensing delay $\tau_{i}$ is larger as shown in Fig. 4. The functional relationship can be written as

$$
\tau_{i}\left(V_{i}\right)=\tau_{0} f\left(V_{i}\right)
$$

where $\tau_{0}$ is the characteristic transit time of the technology (transit time across the channel in the MOS technology or across the base in the bipolar technology).

The time required for the output driver to charge and discharge the large capacitance line by a voltage $V_{i}$ is given by

$$
\begin{aligned}
\tau_{L} & =\frac{C_{L}}{I_{o}} V_{i} \\
\tau_{L} & =\frac{C_{L}}{I_{o}} \frac{1}{G} V_{o}=C_{L} R_{o} \frac{1}{G}=\frac{T_{0}}{G}
\end{aligned}
$$

where

$$
R_{o}=\frac{V_{o}}{I_{o}} \text { and } T_{0}=C_{L} R_{o}
$$

$I_{o}$ represents the current driving capability of the output driver and is given [1] by

$$
I_{o}=C_{D} \frac{V_{o}}{\tau_{\mathrm{Dr}}}
$$

where $C_{D}$ is the input capacitance of the output driver. The minimum possible driver-chain delay, as shown in Section II, is given by

$$
\tau_{\mathrm{ch}}=\tau_{\mathrm{Dr}} e \ln \frac{C_{D}}{C_{G}}
$$

If the input-stage circuit configuration is such that the input voltage $V_{i}$ is sampled and it is then clocked to amplify $V_{i}$ to $V_{o}$, the total delay time $\tau_{D}$ is approximately equal to the driver-chain delay $\tau_{\text {ch }}$ plus the delay associated with driving the large capacitance line $\tau_{L}$ plus the input stage sensing delay $\tau_{i}$

$$
\tau_{D}=\tau_{\mathrm{ch}}+\tau_{L}+\tau_{i}
$$

As shown in Appendix A, the above sum previously given still represents approximately $\tau_{D}$ for other input circuit configurations if $\tau_{L} \geq \tau_{i}$. Substituting in (13), we get

$$
\tau_{D}=\tau_{\mathrm{Dr}} e \ln \frac{C_{D}}{C_{G}}+\frac{C_{L}}{C_{D}} \tau_{\mathrm{Dr}} \frac{V_{i}}{V_{o}}+\tau_{i}\left(V_{i}\right) .
$$

An optimum value of $C_{D}$ results by putting the partial derivative $\left(\partial \tau_{D} / \partial C_{D}\right)=0$, and is given by

$$
C_{D / \mathrm{opt}}=\frac{C_{L}}{e} \frac{V_{i}}{V_{o}}=\frac{C_{L}}{e} \cdot \frac{1}{G} .
$$

Substituting in (13),

$$
\tau_{D}=\tau_{\mathrm{Dr}} e \ln \frac{C_{L}}{C_{G}} \frac{V_{i}}{V_{o}}+\tau_{i}\left(V_{i}\right)
$$

The first term in (16) represents the delay in the driver chain $\tau_{\mathrm{ch}}$ and the output driver $\tau_{L}$ and is less than the delay $\tau_{\mathrm{ch}}$ in (4), as the signal swing on the output lines is reduced by the gain $V_{o} / V_{i}$. Thus, the optimum output driver delay is equal to the delay per stage of the driver chain. The delay times are plotted in Fig. 4 versus $V_{i}$. By equating the partial derivative $\left(\partial \tau_{D} / \partial V_{i}\right)=0$, we get the optimum swing $V_{i \mid \text { min }}$ of the line for minimum delay time $\tau_{D}$

$$
\tau_{\mathrm{Dr}} e \frac{1}{V_{i \mid \min }}+\frac{\partial \tau_{i}}{\partial V_{i}}\left(V_{i \mid \min }\right)=0
$$

which defines $V_{i \mid \text { min }}$ and by substituting in (16) results in the minimum possible delay $\tau_{D \mid \text { min }}$ for transferring a signal on such a high capacitance path.

The input-stage delay $\tau_{i}$ in (6) can be written as a monotonic function of the gain $G$ defined in (5)

$$
\tau_{i}=\tau_{0} f\left(\frac{V_{o}}{G}\right)=\tau_{0} g(G) .
$$

The total delay time in (17) can also be written as a function of the gain $G$

$$
\tau_{D}=\tau_{\mathrm{Dr}} e \ln \frac{C_{L}}{C_{G}} \frac{1}{G}+\tau_{0} g(G) .
$$

The dependence of $\tau_{i},\left(\tau_{\mathrm{ch}}+\tau_{L}\right)$ and $\tau_{D}$ on the gain $G$ is illus. trated in Fig. 4.

If the input-stage circuit configuration is such that

$$
\tau_{i}=\tau_{0} \alpha G^{n}, \quad n \geqslant 1
$$

then (19) reduces to

$$
\tau_{D}=\tau_{\mathrm{Dr}} e \ln \frac{C_{L}}{C_{G}} \frac{1}{G}+\tau_{0} \alpha G^{n} .
$$

Since the frequency bandwidth (BW) of the input sensing stage is proportional to $1 / \tau_{i},(20)$ can be rewritten as

$$
G \cdot \mathrm{BW}=\frac{1}{\tau_{0} \alpha G^{n-1}} .
$$

Thus the input-stage sensing delay $\tau_{i}$ dependence on the gain $G$ is a reformulation of the gain-bandwidth product of the input 

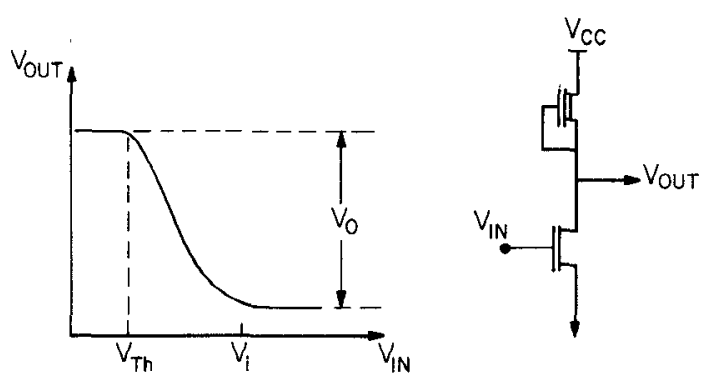

Fig. 5. Depletion-load-inverter input stage.

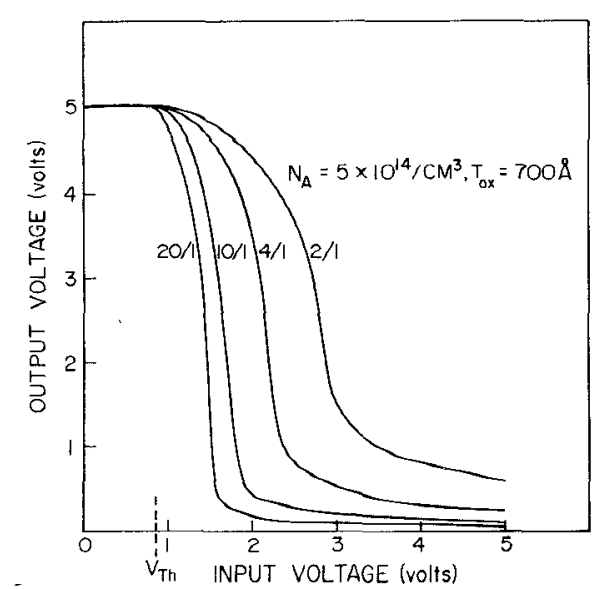

Fig. 6. Input-output voltage characteristics of a depletion-load-inverter input stage for different aspect ratios of the pull-down to pull-up transistors.

stage, which is limited by the characteristic transit time of the technology $\tau_{0}$. The minimum delay time occurs at

$$
\begin{aligned}
G_{\mid \min } & =\left(\frac{\tau_{\mathrm{Dr}_{\mathrm{r}}} e}{n \alpha \tau_{0}}\right)^{1 / n} \quad V_{i \mid \min }=V_{o}\left(\frac{n \alpha \tau_{0}}{e \tau_{\mathrm{Dr}}}\right)^{1 / n} \\
\tau_{i \mid \min } & =\frac{e \tau_{\mathrm{Dr}}}{n}
\end{aligned}
$$

where $\tau_{i \mid \min }$ is independent of the ratio of load capacitance to gate capacitance $\left(C_{L} / C_{G}\right)$. The minimum possible delay $\tau_{D \mid \min }$ for transferring the signal through the high capacitance path is given by

$$
\tau_{D \mid \min }=\tau_{\mathrm{Dr}} e\left[\frac{1}{n}+\ln \frac{C_{L}}{C_{G}}\left(\frac{n \alpha \tau_{0}}{\tau_{\mathrm{Dr}}}\right)^{1 / n}\right] .
$$

We consider below two numerical examples of a single-ended depletion-load-inverter input stage and a differential regenerative strobed-latched input stage.

\section{A. Depletion-Load-Inverter Input Stage}

For the depletion-load MOS inverter input stage in Fig. 5, the input-output characteristics for different aspect ratios are shown in Fig. 6. The gain of the stage is given [2] by

$$
\frac{V_{o}}{V_{i}-V_{\mathrm{th}}}=K \sqrt{r}
$$

where $r$ is the aspect ratios of the load and pull-down transitors and $K$ is a constant given by

$$
K=\frac{2}{\alpha f} \sqrt{V_{Q}+V_{B B}}
$$

where $\alpha$ is the body factor $\left.=\sqrt{\left(2 e N_{A} \epsilon_{s}\right.} / C_{0 x}\right) ; V_{B B}$ is the substate bias; $V_{Q}$ is the quiescent output voltage $\cong\left(V_{c c} / 2\right)$; and $f$ is a constant. The input-stage delay is dominated by the rise time of the stage and is given [1] by

$$
\tau_{i}=4(O+p) \tau_{0}(r+1) \simeq 4(O+p) \tau_{0} r
$$

where

$O$ inverter output fan-out;

$p$ parasitic to intrinsic gate capacitance;

$\tau_{0}$ transit time across gate of pull-down transistor, and is given by

$$
\tau_{0}=\frac{L}{V_{d}} \simeq \frac{L^{2}}{\mu_{\mathrm{eff}}\left(V_{G}-V_{\mathrm{th}}\right)}
$$

where

$$
\begin{array}{ll}
L & \text { gate length of the pull-down transistor; } \\
V_{d} & \text { carrier drift velocity under the gate; } \\
\mu_{\mathrm{eff}} & \text { effective carrier mobility; } \\
\left(V_{G}-V_{\mathrm{th}}\right) & \text { voltage drop across inversion layer in saturation. }
\end{array}
$$

Thus the relationship in (6) reduces to

$$
\tau_{i}=\tau_{0} \frac{E V_{o}^{2}}{\left(V_{i}-V_{\mathrm{th}}\right)^{2}}
$$

where $E$ is a dimensionless constant equal to $\left[4(O+p) / K^{2} \rrbracket\right.$.

Assuming the voltage swing on the high capacitance path is between $V_{\text {th }}$ and $V_{i}$, the delay times for the depletion-loadinverter input stage reduce to

$$
\begin{aligned}
\tau_{\mathrm{ch}}+\tau_{L} & =e \tau_{\operatorname{Dr}} \ln \frac{C_{L}}{C_{G}} \frac{\left(V_{i}-V_{\mathrm{th}}\right)}{V_{o}} \\
\tau_{i} & =\tau_{0} E \frac{V_{o}^{2}}{\left(V_{i}-V_{\mathrm{th}}\right)^{2}} \\
\tau_{D} & =e \tau_{\operatorname{Dr}} \ln \frac{C_{L}}{C_{G}}\left(\frac{V_{i}-V_{\mathrm{th}}}{V_{o}}\right)+\tau_{0} E\left(\frac{V_{o}}{V_{i}-V_{\mathrm{th}}}\right)^{2} .
\end{aligned}
$$

In Fig. $7 \tau_{i},\left(\tau_{\mathrm{ch}}+\tau_{L}\right)$ and $\tau_{D}$ are plotted for a depletionload-inverter input stage with the following parameters: $L=$ $4 \mu \mathrm{m} ; \mu_{\mathrm{eff}} \cong 500 \mathrm{~cm}^{2} / \mathrm{V} \cdot \mathrm{s} ;\left(V_{G}-V_{\mathrm{th}}\right) \cong 4 \mathrm{~V} ; \tau_{0}=1 / 20 \mathrm{~ns}$; $O+p=5 ; k=3$; and $E=2.2$. The driver chain consists of depletion-load push-pull buffers with the following parameters: $P=5, \tau_{0}=1 / 20 \mathrm{~ns} ; r=4 ; \tau_{\mathrm{Dr}}=20 ; \tau_{0}=1 \mathrm{~ns} ; C_{G}=0.1 \mathrm{pF} ;$ $C_{L}=50 \mathrm{pF} ; C_{L} / C_{G}=500 . \tau_{D}$ has a minimum at

$$
\left(V_{i}-V_{\mathrm{th}}\right)_{\min }=W_{o} \sqrt{\frac{2 \tau_{0} E}{e \tau_{\mathrm{Dr}}}}
$$

where the values of $\tau_{i},\left(\tau_{\mathrm{ch}}+\tau_{L}\right), \tau_{0}$ and $G$ are given by

$$
\begin{aligned}
\tau_{i \mid \min } & =0.5 \tau_{\mathrm{Dr}} e \\
\tau_{\mathrm{ch}}+\tau_{L \mid \min } & =e \tau_{\mathrm{Dr}} \ln \frac{C_{L}}{C_{G}} \sqrt{\frac{2 \tau_{0} E}{e \tau_{\mathrm{Dr}}}} \\
\tau_{D \mid \min } & =\tau_{\mathrm{Dr}} e\left[0.5+\ln \frac{C_{L}}{C_{G}} \sqrt{\frac{2 \tau_{0} E}{e \tau_{\mathrm{Dr}}}}\right] \\
G_{\mid \min } & =\sqrt{\frac{e \tau_{\mathrm{Dr}}}{2 \tau_{0} E}} .
\end{aligned}
$$




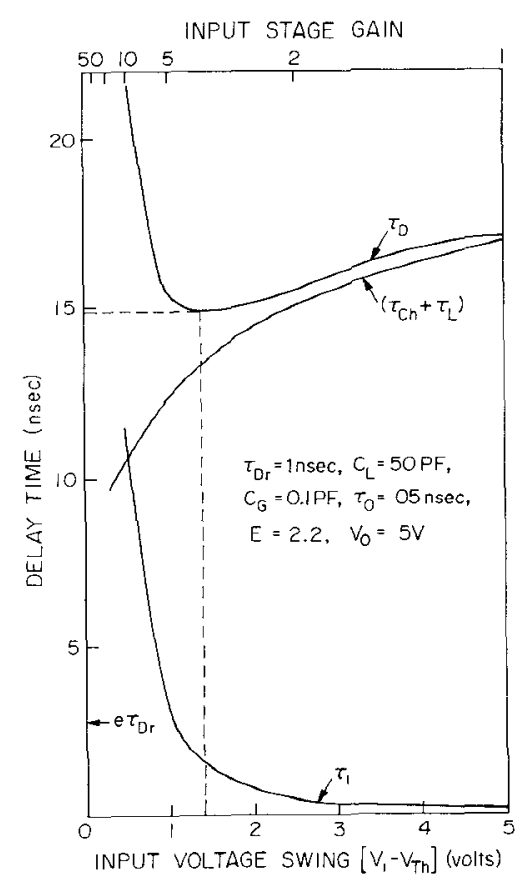

Fig. 7. Delay time versus line voltage swing $V_{i}$ and input-stage gain $G$ for a depletion-load push-pull driver chain driving 50-pF load and a depletion-load-inverter input stage.
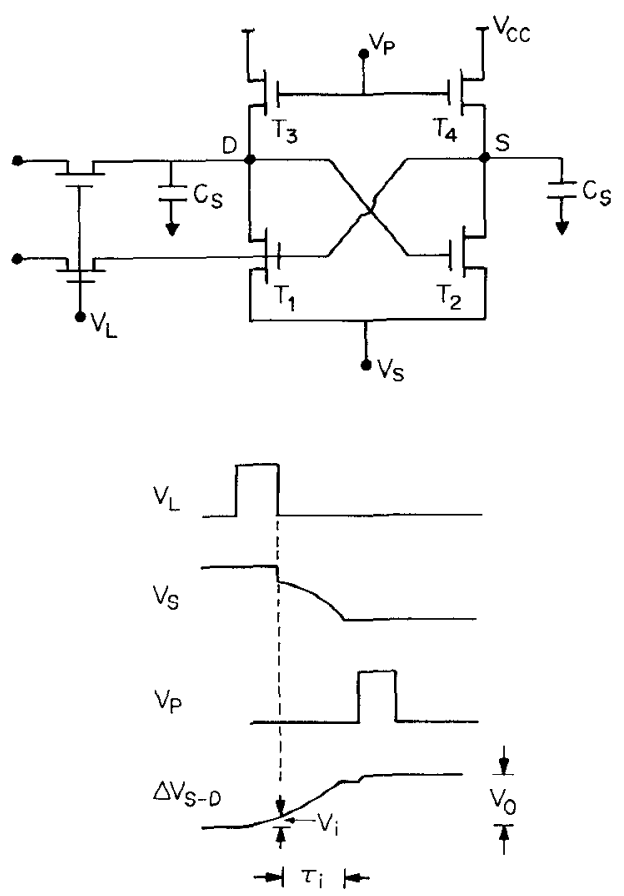

Fig. 8. Regenerative balanced strobed-latch input stage.

In Fig. 7 the delay of the driver chain is the dominant component of the total delay time for input voltage swing $V_{1}>$ $0.8 \mathrm{~V}$ and input-stage gain $G<8$. The total delay time increases logarithmically with the line voltage swing for line voltage swings above the optimum value. The increase in $\tau_{D}$ by increasing the voltage swing on the high capacitance line from 1.4 to $5 \mathrm{~V}$ is only 15 percent. Therefore, in such cases full supply-voltage swing on the high capacitance line provides better immunity against interfering signals with a relatively small delay-time penalty. However, increasing the input-stage

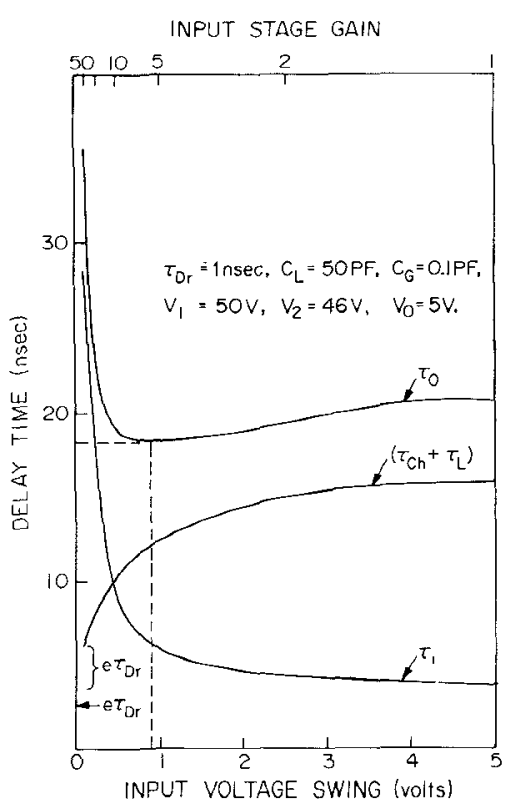

Fig. 9. Delay time versus line voltage swing $V_{i}$ and input-stage gain $G$ for a depletion-load push-pull driver chain driving $50-\mathrm{pF}$ load and a regenerative balanced strobed-latch input stage.

gain by a factor of 3 from the optimum value increases the total delay time $\tau_{D}$ by about 50 percent. Irrespective of the line-to-gate capacitance $C_{L} / C_{G}$, the minimum transmission delay time $\tau_{D}$ on the large capacitance line is achieved with a driver-chain fan-out $f$ equal to $e$ and an input stage of sensing delay $\tau_{i}$, half the delay per stage of the driver chain $\left(e \tau_{\mathrm{Dr}}\right)$.

\section{B. Strobed-Latch Input Stage}

For the strobed-latch input stage in Fig. 8 the latching delay time $\tau_{i}$ is inversely proportional to $V_{i}$ with an optimum latching waveform and no off-side conduction [3] (as shown in Appendix B). The delay times associated with transmission on the high capacitance path with such an input stage are given by

$$
\begin{aligned}
\tau_{i} & =\tau_{0}\left(\frac{V_{1}}{V_{i}}+\frac{V_{2}}{V_{\text {th }}}\right) \\
\left(\tau_{\mathrm{ch}}+\tau_{L}\right) & =e \tau_{\mathrm{Dr}} \ln \frac{C_{L}}{C_{G}} \frac{V_{i}}{V_{o}} \\
\tau_{D} & =e \tau_{\mathrm{Dr}} \ln \frac{C_{L}}{C_{G}} \frac{V_{i}}{V_{o}}+\tau_{0}\left(\frac{V_{1}}{V_{i}}+\frac{V_{2}}{V_{\mathrm{th}}}\right) .
\end{aligned}
$$

The delay times are plotted in Fig. 9 for a strobed input latch with the following parameters:

$$
\begin{aligned}
& C_{S}=0.1 \mathrm{pF} \quad C_{g}=0.03 \mathrm{pF} \quad \frac{W}{L}=\frac{15}{5} \quad \frac{W_{e l}}{L_{e l}}=\frac{12}{3.8} \\
& f=0.77 \quad t_{0 x}=700 \AA \quad p=12.6 \times 10^{-6} \mathrm{~A} / \mathrm{V}^{2} \\
& \tau_{0}=0.05 \mathrm{~ns} \quad V_{1}=50 \mathrm{~V} \quad V_{\text {th }}=0.7 \mathrm{~V} \quad V_{2}=46 \mathrm{~V} \\
& V_{o}=5 \mathrm{~V} \quad \tau_{\mathrm{Dr}}=1 \mathrm{~ns} \quad C_{L}=50 \mathrm{pF} \quad C_{G}=0.1 \mathrm{pF} .
\end{aligned}
$$

The total delay time has a minimum at

$$
V_{i / \mathrm{m} ı \mathrm{n}}=\frac{\tau_{0}}{e \tau_{\mathrm{Dr}}} V_{1}
$$


where the delay times are given by

$$
\begin{aligned}
& \tau_{i \mid \min }=e \tau_{\mathrm{Dr}}+\tau_{0} \frac{V_{2}}{V_{\mathrm{th}}} \\
&\left(\tau_{\mathrm{ch}}+\tau_{L}\right)_{\min }=e \tau_{\mathrm{Dr}} \ln \frac{C_{L}}{C_{G}} \frac{\tau_{0}}{e \tau_{\mathrm{Dr}}} \cdot \frac{V_{1}}{V_{o}} \\
& \tau_{D \mid \min }=e \tau_{\mathrm{Dr}}\left[1+\ln \left(\frac{C_{L}}{C_{G}} \frac{\tau_{0}}{e \tau_{\mathrm{Dr}}} \frac{V_{1}}{V_{o}}\right)\right. \\
&\left.+\frac{\tau_{0}}{e \tau_{\mathrm{Dr}}} \frac{V_{2}}{V_{\mathrm{th}}}\right] \\
& G_{\mid \min }= \frac{V_{o}}{V_{i}}=\frac{V_{o}}{V_{1}} \frac{e \tau_{\mathrm{Dr}}}{\tau_{0}}
\end{aligned}
$$

Similarly, in this case the increase in the total delay time $\tau_{D}$ for a voltage swing on the high capacitance path larger than the optimum value is rather small. Also, minimum transmission delay $\tau_{D}$ across the high capacitance path is achieved with a sensing delay $\tau_{i}$ of the input stage comparable to the delay per stage of the driver chain $e \tau_{\mathrm{Dr}}$.

\section{Sensing Signals on Large Capacitance Lines}

In many cases, the driver circuit at one end of the line is limited by constraints that limit the driver optimization previously discussed. Such cases are often encountered in sensing signals from small cells of large memory arrays. We consider below how we may minimize the total delay time of signal transmission on a high capacitance path with a fixed drive source at one end by optimizing the gain of the input stage and the line voltage swing.

In Fig. 10, the output driver is represented by a fixed current source $I_{o}$ which drives the large capacitance line $C_{L}$ with a voltage swing equal to $V_{i}$. The input stage senses the input signal at the other end of the line and generates an output voltage $V_{o}$ equal to the supply voltage. The total transmission delay time $\tau_{D}$ in this case is equal to the sum of the sensing delay time $\tau_{i}$ of the input stage and the line delay time $\tau_{L}$ associated with the charging and discharging of the line capacitance $C_{L}$. Using (7) and (9), the delay time $\tau_{D}$ is given by

$$
\tau_{D}=\tau_{0} f\left(V_{i}\right)+T_{o} \frac{V_{i}}{V_{o}}
$$

If the input-stage delay $\tau_{i}$ is given by (20), the minimum delay time occurs at

$$
\begin{aligned}
G_{\mid \min } & =\left[\frac{\tau_{0}}{n \alpha \tau_{0}}\right]^{1 /(n+1)} \\
V_{i \mid \min } & =V_{o}\left[\frac{n \alpha \tau_{0}}{\tau_{0}}\right]^{1 /(n+1)} \\
\tau_{i \mid \min } & =\frac{\tau_{L \mid \min }}{n}
\end{aligned}
$$

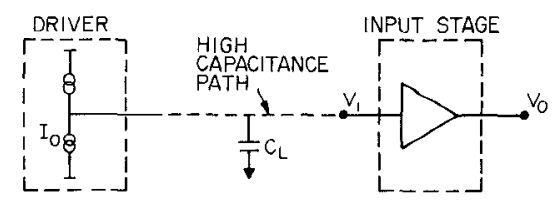

Fig. 10. Fixed driver drives a high capacitance load $C_{L}$. Input stage receives input signal $V_{i}$ to generate output yoltage $V_{O}$.

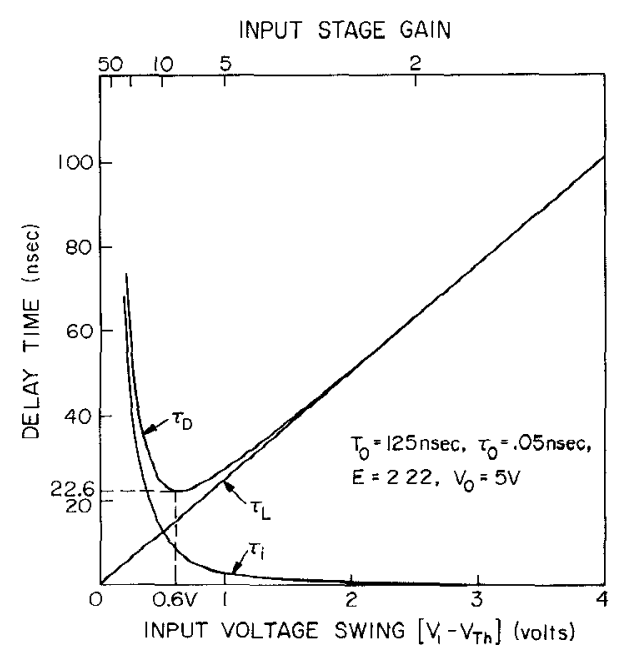

Fig. 11. Delay times versus line voltage swing $V_{i}$ and input-stage gain $G$ for a fixed driver driving a large capacitance load with a drive-time constant $T_{0}=125 \mathrm{~ns}$, and a depletion-load-inverter input stage.

The minimum delay time $\tau_{D \mid \text { min }}$ for transferring the signal across the high capacity path is given by

$$
\begin{aligned}
\tau_{D \mid \min } & =\left[1+\frac{1}{n}\right] \tau_{L \mid \min } \\
& =\left[1+\frac{1}{n}\right] \tau_{0}\left[\frac{\tau_{0} \alpha n}{\tau_{0}}\right]^{1 /(n+1)} .
\end{aligned}
$$

We consider below two numerical examples for a singleended depletion-load-inverter input stage and a differential regenerative strobed-latch input stage.

\section{A. Depletion-Load-Inverter-Input Stage}

For the depletion-load-inverter input stage in Fig. 6" the input delay time is given by

$$
\tau_{i}=\tau_{0} E \frac{V_{o}^{2}}{\left(V_{i}-V_{\mathrm{th}}\right)^{2}} .
$$

Assuming the voltage swing on the high capacitance line $C_{L}$ is between $V_{i}$ and $V_{\mathrm{th}}$, the total delay time $\tau_{D}$ reduces to

$$
\tau_{D}=\tau_{0} E \frac{V_{o}^{2}}{\left(V_{i}-V_{\mathrm{th}}\right)^{2}}+T_{o} \frac{\left(V_{i}-V_{\mathrm{th}}\right)}{V_{o}} .
$$

In Fig. $11, \tau_{i}, \tau_{L}$, and $\tau_{D}$ are plotted for the following parameters:

$$
\begin{aligned}
& L=4 \mu \mathrm{m} \quad \mu_{\mathrm{eff}}=500 \mathrm{~cm}^{2} / \mathrm{V} \cdot \mathrm{s} \quad\left(V_{o}-V_{\mathrm{th}}\right)=4 \mathrm{~V} \\
& \tau_{0}=1 / 20 \mathrm{~ns} \quad E=2.2 \quad C_{L}=1 \mathrm{pF} \quad I_{o}=40 \mu \mathrm{A} \\
& T_{o}=125 \mathrm{~ns} .
\end{aligned}
$$


$\tau_{D}$ has a minimum at

$$
\left(V_{i}-V_{\mathrm{th}}\right)=\left[\frac{2 \tau_{0} E}{T_{o}}\right]^{1 / 3} V_{o}
$$

where the values of $\tau_{i}, \tau_{L}, \tau_{D}$, and $G$ are given by

$$
\begin{aligned}
\tau_{i \mid \min } & =\tau_{0}\left[\left(\frac{T_{o}}{2 \tau_{0}}\right)^{2} E\right]^{1 / 3} \\
\tau_{L / \min } & =2 \tau_{i \mid \min } \\
\tau_{D \mid \min } & =3 \tau_{i \mid \min } \\
G_{\mid \min } & =\left[\frac{T_{o}}{2 \tau_{0} E}\right]^{1 / 3} .
\end{aligned}
$$

In Fig. 11 , a minimum of $\tau_{D}$ at 22 ns exists at an input voltage swing of $0.6 \mathrm{~V}$, which is about a factor of 5 less than the delay time $\tau_{D}$ with full supply voltage swing. The minimum delay time is achieved with the input-stage delay time $\tau_{i}$ equal to half the line delay time $\tau_{L}$.

\section{B. Strobed-Latch Input Stage}

For the strobed-latch input stage with an optimum latching waveform and no off-side conduction, the total delay time is given by

$$
\tau_{D}=\tau_{0}\left(\frac{V_{1}}{V_{i}}+\frac{V_{i}}{V_{\mathrm{th}}}\right)+T_{o} \frac{V_{i}}{V_{o}} .
$$

The delay times are plotted in Fig. 12 for a strobed input latch with the following parameters:

$$
\begin{array}{llll}
C_{S}=0.1 \mathrm{pF} \quad C_{g}=0.03 \mathrm{pF} \quad \frac{W}{L}=\frac{15}{5} & \frac{W_{e l}}{L_{e l}}=\frac{12}{3.8} \\
f=0.77 \quad t_{0 x}=700 \AA \quad \tau_{0}=0.05 \mathrm{~ns} & V_{1}=50 \mathrm{~V} \\
V_{\text {th }}=0.7 \mathrm{~V}, \quad V_{2}=46 \mathrm{~V} \quad V_{o}=5 \mathrm{~V} & C_{L}=1 \mathrm{pF} \\
I_{o}=40 \mu \mathrm{A} & T_{o}=125 \mathrm{~ns} .
\end{array}
$$

$\tau_{D}$ has a minimum at

$$
V_{i \mid \min }=\sqrt{\frac{\tau_{0}}{T_{0}}} V_{o} V_{1}
$$

where the values of $\tau_{i}, \tau_{L}, \tau_{D}$, and $G$ are given by

$$
\begin{aligned}
\tau_{i \mid \min } & =\tau_{0}\left[\sqrt{\frac{T_{0}}{\tau_{0}} \frac{V_{1}}{V_{o}}}+\frac{V_{\mathrm{th}}}{V_{2}}\right] \\
\tau_{L \mid \min } & =\tau_{0} \sqrt{\frac{T_{0}}{\tau_{0}} \frac{V_{1}}{V_{o}}} \\
\tau_{D \mid \min } & =\tau_{0}\left[2 \sqrt{\frac{T_{0}}{\tau_{0}} \frac{V_{1}}{V_{o}}}+\frac{V_{\mathrm{th}}}{V_{2}}\right] \\
G_{\mid \min } & =\sqrt{\frac{\tau_{0}}{T_{0}} \frac{V_{1}}{V_{o}}} .
\end{aligned}
$$

In Fig 12 the minimum delay time $\tau_{D}$ is $18.5 \mathrm{~ns}$ for $V_{i}=0.3 \mathrm{~V}$. Similarly, in this case the minimum total delay time to transmit the signal across the large capacitance line is achieved with a line voltage swing and input-stage gain such that the sensing

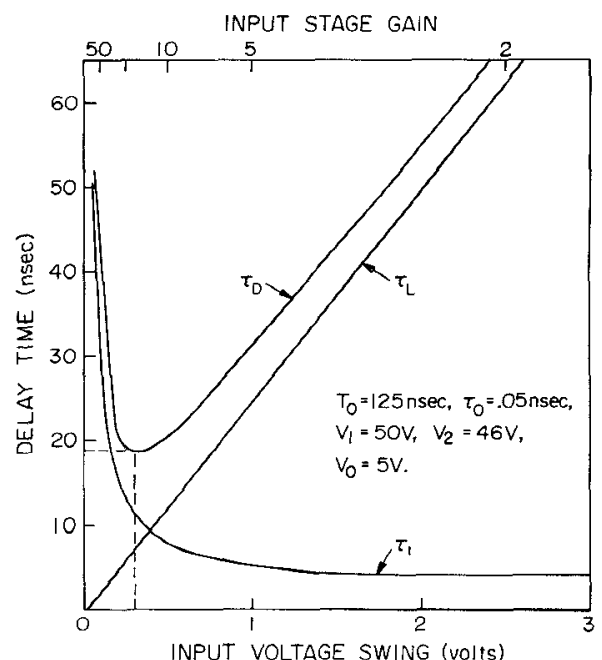

Fig. 12. Delay time versus line voltage swing $V_{i}$ and input-stage gain $G$ for a fixed driver driving a large capacitance load with a drive-time constant $T_{0}=125 \mathrm{~ns}$ and a regenerative balanced strobed-latch input stage.

delay $\tau_{i}$ of the input stage is comparable to the line delay time $\tau_{L}$.

\section{Conclusions}

We have examined how to minimize the delay time associated with the transmission of signals across large capacitance paths by optimizing the driving and sensing circuits. In our analysis we have considered the design of the driver and sensing circuits in general terms by optimizing the fan-out of the driver-stages chain, the gain of the input sensing circuit, and the path voltage swing.

For driving large capacitive loads, we have found that the drive delay time of a chain of successive drivers has a broad minimum at a fan-out factor $f$ around $e$, the base of the natural logarithms. The delay times of each stage of the driver chain are equal to $e \tau_{\mathrm{Dr}}$, where $\tau_{\mathrm{Dr}}$ is the delay time of a driver driving a similar driver. This is a result of the exponential growth of the drive capabilities of the successive stages of the driver chain. At this minimum, the number of stages in the driver chain is equal to the natural logarithm of the load capacitance to the gate capacitance $C_{L} / C_{G}$. The minimum driver-chain delay time $\tau_{\mathrm{ch}}$ is equal to the delay per stage of the driver chain $e \tau_{\mathrm{Dr}}$ times the number of stages $\ln \left(C_{L} / C_{G}\right)$. For fan-out factor $f$ larger than $e$, the relative delay time penalty is relatively small.

Minimization of the total transmission time on a large capacitance path, in cases where the fan-out factor of the driver chain, the gain of the input sensing stage, and the path voltage swing can be optimized, have been examined. Minimum total delay is achieved with a driver chain of fan-out $f$ equal to $e$ and an input stage with an input sensing delay related to the delay per stage of the driver chain according to the delay-time gain characteristic of the input stage. Irrespective of the ratio of the path-to-gate capacitance, the total delay time has a broad minimum for line voltage swings above the optimum swing, but a rather sharp minimum for input-stage gain above the optimum gain. For line voltage swings above the optimum value, the driver chain and line delay times are dominant and 
the total delay times increase logarithmically with the line voltage swing. Therefore, in such cases full supply voltage swing on the high capacitance line provides better noise immunity against interferring signals with a relatively small time penalty. Delay times for push-pull depletion-load-driver stages with a single-ended depletion-load-inverter input stage and with a balanced regenerative strobed latch have been analyzed. For a single-ended depletion-load-inverter input stage (delay time $\tau_{i} \alpha^{a l} 1 /(\text { gain })^{2}$ ), the minimum total delay is achieved with an input-stage delay $\tau_{i}$ equal to one-half the delay per stage of the driver chain $e \tau_{\mathrm{Dr}}$. For a regenerative balanced strobed. latch input stage (delay time $\tau_{i} \alpha^{a l} 1 /$ (gain)), minimum delay time occurs when the input-stage delay $\tau_{i}$ is comparable to the delay per stage of the driver chain.

Minimization of the total transmission time on a large capacitance path in cases where the driver is fixed and the line voltage swing and the gain of the input stage can be optimized, have been presented. Cases of fixed drivers of large capacitance lines are encountered in sensing stored information from memory cells of large arrays. Minimum total delay is achieved with a line voltage swing and an input stage such that the line delay time is related to the input sensing delay according to the delay-time gain characteristics of the input stage. For a single-ended depletion-load-inverter input stage (delay time $\tau_{i} \alpha^{a l} 1 /(\text { gain })^{2}$ ), the minimum total delay is achieved at a line voltage swing and input-stage gain such that the input-stage delay time $\tau_{i}$ is half the line delay time $\tau_{L}$. For a differential regenerative balanced strobed-latch input stage (delay time $\tau_{i} \alpha^{a l} 1 /($ gain $)$ ), the minimum total delay occurs when the input-stage delay $\tau_{i}$ is comparable to the line delay time $\tau_{L}$. Deviations of the gain and line voltage swing by a factor of 2 from the minimum may increase the total delay time by as much as 75 percent for the examples considered in this paper.

In general, we may conclude that a minimum transmission time of signals in a system consisting of several stages is achieved when the delay times of the different stages are comparable. For the case of driving and sensing signals from large capacitance paths, minimum delay time is achieved when the delay times of the successive stages of the driver chain, the high capacitance path, and the input sensing stage are comparable.

\section{APPENDIX A}

The output of the input-stage circuit can be represented by a source voltage $V_{s}$ that corresponds to the amplified undelayed input voltage $V_{i}$ to the stage and a delay $\tau_{i}$ provided by a simple $R C$ circuit as shown in Fig. 13(a). We show below that if the input-stage delay $\tau_{i}$ is less than the line delay $\tau_{L}$, the total delay $\tau_{D}$ is approximately equal to the sum of the input stage delay $\tau_{i}$ and the line delay $\tau_{L}$.

In Fig. 13(b) the responses of the input-stage equivalent circuit to a step, a ramp, and a sinusoidal input are shown. For a step input

$$
t>0 \quad V_{S}=V_{0} \quad V_{\text {out }}=V_{0}\left[1-\exp \left(-t / \tau_{i}\right)\right] .
$$

For a ramp input

$$
t>0 \quad V_{S}=\frac{t}{\tau_{L}} V_{0} \quad V_{\text {out }}=\frac{\left(t-\tau_{i}\right)}{\tau_{L}}+\frac{V_{0}}{\tau_{L}} \exp \left(-t / \tau_{i}\right) .
$$

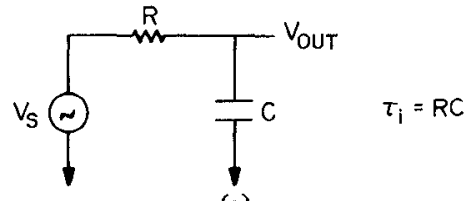

(a)
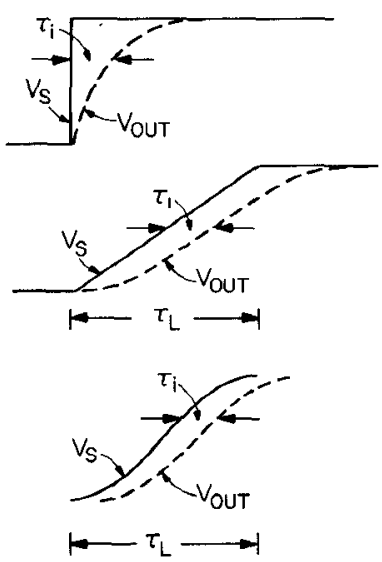

(b)

Fig. 13. (a) Equivalent circuit of the input sensing stage. (b) The output responses of the input stage to a step, a ramp, and a sinusoidal input.

$$
\begin{aligned}
& \text { If } \tau_{L}>\tau_{i}, \text { then } \\
& V_{\text {out }} \simeq \frac{\left(t-\tau_{i}\right) V_{0}}{\tau_{i}} .
\end{aligned}
$$

For a sinusoidal input

$$
\begin{gathered}
t>0 \quad V_{S}=\frac{V_{0}}{2}\left[1-\cos \frac{\pi t}{\tau_{L}}\right] \quad V_{\text {out }}=\frac{V_{0}}{2} \\
.\left[1-\cos \frac{\pi(t-\theta)}{\tau_{2}}\right] \theta=\frac{\tau_{L}}{\pi} \tan ^{-1} \frac{\pi \tau_{i}}{\tau_{L}} .
\end{gathered}
$$

If $\tau_{L}>\tau_{i}$, then

$$
V_{\mathrm{out}} \simeq \frac{V_{0}}{2}\left[1-\cos \frac{\pi\left(t-\tau_{i}\right)}{\tau_{L}}\right]
$$

From (A-3) and (A-4), it is apparent that for $\tau_{L}>\tau_{i}$ the output voltage $V_{\text {out }}$ is delayed from the amplified undelayed input voltage $V_{s}$ by the input stage delay $\tau_{i}$. Thus, in general, if $\tau_{i}<\tau_{L}$, the total delay $\tau_{D}$ is the sum of the line delay $\tau_{L}$ and the input-stage delay $\tau_{i}$

$$
\tau_{D} \simeq \tau_{L}+\tau_{i}
$$

\section{APPENDix B}

Dynamic MOS regenerative latch sensors, as in Fig. 8, can amplify a small initial imbalance $V_{i}$ between the two internal nodes $D$ and $G$ to a voltage difference comparable to the initial power-supply voltage $V_{0}$. For smaller initial voltage imbalance the latch-up time is, in general, larger. For any given initial imbalance $V_{i}$, there is an ideal latching waveform that minimizes the latch time [3]. The initial imbalance represents the sum of the real voltage imbalance and any threshold imbalance of the MOS crosscoupled transistor pair. The general shape of the optimum latching waveform is shown in Fig. 8. It con- 
sists of an initial step followed by a ramp of gradually increasing slope to the final voltage value.

The internal latch nodes $D$ and $G$ are precharged to $V_{0}$. The input voltage introduces an imbalance $V_{i}$ on nodes $D$ and $G$. To minimize threshold imbalances and reduce power dissipation, the flip-flop load devices are turned off during latchup. The latch-up waveform $V_{s}(t)$ can be selected such that no current flows through the off-side during latchup to maximize the final latched imbalance. However, coupling capacitances to the off-side lower its final voltage and lowers the conduction of the on transistor, thus increasing the latching time. The optimum latching waveform [3] consists of two portions given by

$$
V_{s}(t)=V_{0}-V_{\text {th }}-\frac{V_{i}-t / \tau}{1-t / \tau} \text { for } t<t_{\text {sat }}
$$

and

$$
\begin{array}{r}
V_{s}(t)=V_{0}+V_{\text {in }}-V_{\text {th }}-\frac{V_{\text {th }}}{2 f}\left\{3+\exp \left[\left(t-t_{\text {sat }}\right) / \tau_{1}\right]\right\} \\
\text { for } t>t_{\text {sat }}
\end{array}
$$

where

$$
\begin{aligned}
f & =\frac{C_{s}}{\left(C_{s}+C_{g}\right)} \\
\tau & =\frac{2 C_{s}}{\beta f^{2} V_{i}} \\
\tau_{1} & =\frac{C_{s}}{\beta f V_{\text {th }}} \\
t_{\text {sat }} & =\frac{2 C_{s}\left(V_{\text {th }}-f V_{i}\right)}{\beta f^{2} V_{\text {th }} V_{i}}
\end{aligned}
$$

where $C_{g}, \beta$, and $V_{\text {th }}$ are the gate capacitance, current factors, and threshold voltages of $T_{1}$ and $T_{2}$; and $C_{S}$ is the capacitance of latch nodes $D$ and $G$. The latch time $\tau_{i}$ is given by

$$
\tau_{i}=\tau_{0}\left(\frac{V_{1}}{V_{i}}+\frac{V_{2}}{V_{\mathrm{th}}}\right)
$$

where $\tau_{0}$ is the transit time across gate and is given by

$$
\begin{aligned}
\tau_{0} & =\frac{C_{g}}{\beta\left(V_{G}-V_{\mathrm{th}}\right)}=\frac{L^{2}}{\mu\left(V_{G}-V_{\mathrm{th}}\right)} \\
V_{1} & =\frac{2 C_{s}}{C_{g}} \frac{\left(V_{\mathrm{th}}-f V_{i}\right)}{V_{\mathrm{th}}} \frac{V_{0}}{f^{2}} \\
V_{2} & =\frac{C_{s}}{C_{g}} \frac{V_{0}}{f} \ln \left[\frac{f\left(V_{0} \pm V_{i}-V_{\mathrm{th}}\right)-V_{\mathrm{th}} / 2}{V_{\mathrm{th}} / 2}\right] .
\end{aligned}
$$

Thus the total latch $\tau_{i}$ is approximately inversely proportional to the initial unbalance $V_{i}$.

\section{REFERENCES}

[1] C. A. Mead and L. A. Conway, Introduction to VLSI Systems, Limited Printing, 1978.

[2] D. Senderowicz, D. A. Hodges, and P. Gray, "High-performance NMOS operational amplifier," IEEE J. Solid-State Circuits, vol. SC-13, pp. 760-766, Dec. 1978.

[3] W. T. Lynch and H. J. Boll, "Optimization of the patching pulse for dynamic flip-flop sensors," IEEE J. Solid-State Circuits, vol. SC-9, pp. 49-55, Apr. 1974.

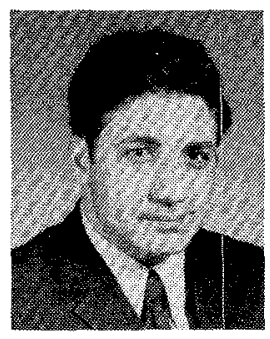

Amr M. Mohsen (S'72-M'74) received the B.Sc. degree in electrical engineering from Cairo University, Cairo, Egypt, in 1968, the M.S. degree in solid-state science and material engineering from the American University in Cairo, Egypt, in 1970, the M.S. degree in electrical engineering and the Ph.D. degree in electrical engineering and applied physics, both from the California Institute of Technology, Pasadena, in 1971 and 1973, respectively.

From 1968 to 1970 he served as an Instructor in the Department of Electrical Engineering, Cairo University. From 1970 to 1973 he was a Research Assistant in the Department of Electrical Engineering at the California Institute of Technology and served as a Consultant in the area of charge-coupled devices and MOS integrated circuits in semiconductor industrial corporations. In 1973 he joined Bell Telephone Laboratories at Murray Hill, NJ, as a Member of the Technical Staff, where he worked on the development of chargecoupled devices. In 1975 he left Bell Telephone Laboratories to participate in founding Mnemonics, where he was Manager of Component Design. He is currently with Intel Corporation, Santa Clara, CA, and on the faculty of the California Institute of Technology, Pasadena. His interests are in the areas of MOS devices and circuits development for VLSI. He has authored and coauthored over 30 technical articles and has several patents granted and pending.

Dr. Mohsen is a member of the American Physical Society and Sigma $\mathrm{Xi}$.

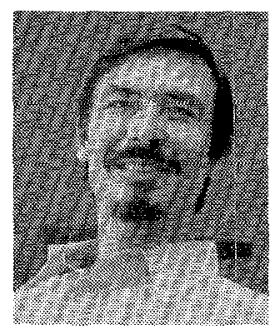

Carver A. Mead received the B.S. degree in 1956, the M.S. degree in 1957, and the Ph.D. degree, in 1957, all from the California Institute of Technology, Pasadena.

He has been a member of the faculty of that institution since 1957. His research has contributed to the understanding of tunneling in solids, current-flow mechanisms in thin dielectric films, metal-semiconductor barriers, band energies in semiconductors, and electronic processes in insulators. He has proposed and demonstrated the operation of a number of new solid-state electronic devices and holds several U.S. patents.

Dr. Mead is a fellow of the American Physical Society and is a member of Sigma Xi. 